\title{
Tuning a synthetic in vitro oscillator using control-theoretic tools
}

\author{
Christopher Sturk, Elisa Franco and Richard M. Murray
}

\begin{abstract}
This paper demonstrates the effectiveness of simple control-theoretic tools in generating simulation-guided experiments on a synthetic in vitro oscillator. A theoretical analysis of the behavior of such system is motivated by high cost, time consuming experiments, together with the excessive number of tuning parameters. A simplified model of the synthetic oscillator is chosen to capture only its essential features. The model is analyzed using the small gain theorem and the theory of describing functions. Such analysis reveals what are the parameters that primarily determine when the system can admit stable oscillations. Experimental verification of the theoretical and numerical findings is carried out and confirms the predicted results regarding the role of production and degradation rates.
\end{abstract}

\section{INTRODUCTION}

Synthetic biology has two main objectives: engineering new biological systems out of characterized parts, and, by systematic modification of existing systems, improving our understanding of design principles. This interdisciplinary field attracts scientists from the areas of biology, mathematics, physics and engineering: a strong theoretical analysis of experimental data increases our ability to interpret and predict the behavior of engineered biological devices.

When operating in an in vitro environment with a limited number of biological parts, scientists have the opportunity to program and deeply investigate the molecular interactions that produce overall designed behaviors. This is one of the most important features of the field of DNA nanotechnology [12], and is a key to the development of molecular computation [11]. But even in a controlled environment, there are cases in which the behavior of the system under observation needs a thorough theoretical and experimental analysis to be correctly interpreted.

A recently proposed synthetic in vitro oscillator [5], composed only of nucleic acids and two enzyme species, presents several challenges regarding its dynamics and tuning. In particular, detailed modeling of the underlying chemical reaction network offers a poor qualitative understanding of its behavior: the complex model is in turn of little help in aiding experiments to modulate the frequency/amplitude characteristics of the circuit. Additionally, the cost and duration of the experiments make it well worth looking for a better modeling

Christopher Sturk is with the Department of Automatic Control, Royal Institute of Technology (KTH), Osquldas väg 10, SE-100 44 Stockholm, Sweden. chsturk@kth. se

Elisa Franco and Richard M. Murray are with the Division of Engineering and Applied Sciences, California Institute of Technology, Pasadena, CA 91125. elisafdcaltech. edu, murraydcds. caltech.edu

The authors would like to thank Erik Winfree for helpful discussions and advise regarding the design and synthesis of in vitro genetic circuits and the Department of Automatic Control LTH, Lund University, Sweden for their support. resource that could qualitatively predict the features of the oscillator.

In this paper we consider a simplified model of the mentioned synthetic oscillator [7] and study its characteristics using classical control-theoretic tools rather than solely simulations of its differential equations. In particular, we used the small gain theorem for monotone systems [2], [1] to derive parametric conditions for which the system can admit an oscillatory regime. Such results are numerically refined using the method of the describing functions, which is an appropriate tool for systems presenting static nonlinearities and delays.

Under certain simplifying assumptions, we found that RNA production and degradation, together with the Hill functions thresholds, confine the region of the parameter space where oscillations are achievable. We focused on the role of production and degradation, mapping it qualitatively to the amounts of enzymes used in the experiments. By varying the production/degradation enzyme ratio and total enzyme volume, we collected data that confirm the main features of the model predictions. The theoretical and numerical analysis were therefore useful in guiding the experimental choices and allowed us to obtain a tuning methodology.

The paper is organized as follows. The synthetic oscillator we consider is described in Section II, where we outline its biological features and introduce the chosen simplified mathematical model. In Section III we report the smallgain theorem analysis and the numerical results based on the describing function method. Finally, experimental results are reported in Section IV.

\section{A SYNTHETIC in vitro OSCILLATOR}

The in vitro genetic circuits considered in this paper consist only of nucleic acids (DNA and RNA) and two enzyme species, RNA polymerase (RNAP) and RNase H. RNAP binds to DNA double-stranded promoter regions and transcribes the downstream sequence into RNA. The RNase $\mathrm{H}$ instead hydrolyses and degrades RNA in RNA-DNA duplexes, releasing the DNA strand.

The genes are synthetic and can be designed using existing software packages [8], [9]. Each gene state can be switched on and off by designing its promoter with a nick on the coding template (the strand complement-transcribed by RNAP). Exploiting the mechanism of toehold-mediated branch migration [13], the promoter can be covered or uncovered, effectively reducing the rate of transcription by RNAP. For example: a synthetic gene is in an off state when the promoter is incomplete, i.e. partly single stranded. A promoter-complementary activating DNA strand in solution 


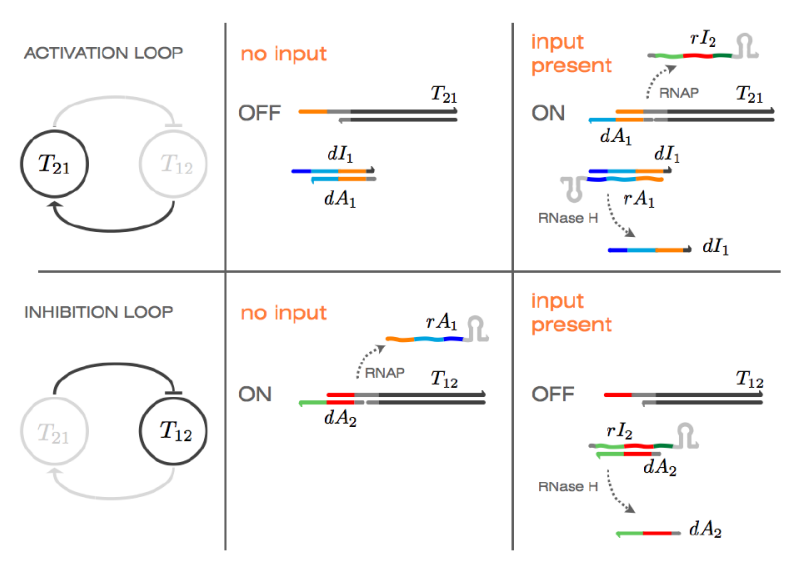

Fig. 1: Scheme representing the main chemical reactions occurring in the considered biochemical oscillator. This is a two-node oscillator: the synthetic genes $T_{12}$ and $T_{12}$ respectively repress or activate one another. Single stranded nucleic acids $d A_{1}, d A_{2}$ and $d I_{1}$ are DNA species whose concentration is controlled; $r A_{1}$ and $r I_{2}$ are RNA species. Arrows on the nucleic acid strands indicate the $5^{\prime}$ to $3^{\prime}$ orientation. The molecular domains are assigned a color to emphasize their different roles. Areas that are complementary have the same color. In orange and red we indicate the activation/inhibition domains, while the branch migration initiation toeholds are colored in blue, cyan and light green. Gray and dark gray indicate promoter and transcribed regions.

will switch the gene on. This activator is designed to present an exposed overhang, i.e. is longer than the promoter binding region. An inhibiting strand fully complementary to the activator can strip it off from the promoter, switching the gene off. While activators can only be designed out of DNA, inhibitors can be RNA molecules. Different genes can therefore be interconnected.

The circuit considered in this paper consists of two synthetic genes connected by the RNA they transcribe, as shown in Figure 1. The gene $T_{21}$ transcribes RNA species $r I_{2}$ which inhibits gene $T_{12}$. The latter in turn produces $r A_{1}$, which activates $T_{21}$. Through this interconnection, experiments on this circuit show an oscillatory behavior.

The full dynamics of this biochemical oscillator can be derived by the underlying chemical reactions. Such a model presented in Chapter 5 of [5] consists of 7 differential equations and over 20 parameters. A simplified model capturing the qualitative behavior of the system [7] that is more amenable to analysis is:

$$
\begin{gathered}
\frac{d\left[r A_{1}\right](t)}{d t}=k_{p}\left[T_{12} A_{2}\right](t)-k_{d}\left[r A_{1}\right](t), \\
\frac{d\left[r I_{2}\right](t)}{d t}=k_{p}\left[T_{21} A_{1}\right](t)-k_{d}\left[r I_{2}\right](t),
\end{gathered}
$$

where

$$
\begin{aligned}
& {\left[T_{12} A_{2}\right](t)=\left[T_{12}^{t o t}\right] \frac{K_{l}^{n}}{K_{1}^{n}+\left[r I_{2}\right](t-\tau)^{n}},} \\
& {\left[T_{21} A_{1}\right](t)=\left[T_{21}^{t o t}\right] \frac{\left[r A_{1}\right](t-\tau)^{n}}{K_{A}^{n}+\left[r A_{1}\right](t-\tau)^{n}} .}
\end{aligned}
$$

The model assumes a rate of transcription proportional to the concentration of the activated template and a rate of degradation proportional to the concentration of RNA. The constants $k_{p}$ and $k_{d}$ can be thought of as the concentration of RNAP and RNase $\mathrm{H}$ respectively. The activation of the two templates is described by Hill functions with a delay. The DNA activator $d A_{2}$ and inhibitor $d I_{1}$ act as thresholds for $T_{12}$ and $T_{21}$ respectively: the Hill apparent dissociation constants $K_{I}$ and $K_{A}$ represent the concentration of input RNA that is needed before the switches start turning on. The overall hybridization dynamics, which may be affected by unwanted and unknown reactions, are accounted for in the delay $\tau$.

In the rest of the paper, unless otherwise stated, the parameters will be set to, $\left[T_{12}^{\text {tot }}\right]=\left[T_{21}^{\text {tot }}\right]=100 \mathrm{nM}$, $K_{A}=K_{I}=1 \mu \mathrm{M}, k_{p}=0.04 s^{-1}, k_{d}=0.002 \mathrm{~s}^{-1}$, $n=5, \tau=180 \mathrm{~s}$. With this choice, the system defined by equations (1)-(4), will display sinusoidal oscillations with a period of about 30 minutes. This choice of the parameters was initially made for consistency with [7].

\section{CONTROL THEORETIC ANALYSIS}

The small gain theorem for monotone systems is useful to explore the areas of the parameter space of the synthetic oscillator which admit an oscillatory regime. This method can be used to prove the existence of global asymptotic stability, which rules out the existence of stable oscillations. In the following section we will present a closed form expression for when the dynamical system converges to an equilibrium regardless of the initial state. Furthermore the describing function technique will be applied numerically to verify when stable oscillations are achieved.

\section{A. The small gain theorem for monotone systems}

The small gain theorem for monotone systems [2] can be used to determine when a monotone system is globally asymptotically stable. Let us briefly recall the definition of a monotone system. Consider the following I/O system:

$$
\frac{d x}{d t}=f(x(t), u(t)) \quad y=h(x),
$$

where $x$ represents the state vector, $u$ and $y$ are scalar input and output respectively (only SISO systems are considered in this paper). Denote the solution to the initial value problem (5), with $x(0)=x_{0}$, as $\varphi\left(t, x_{0}, u\right)$. Let $x(t)$, $u(t)$ and $y(t)$ evolve on the sets $X, U$ and $Y$ respectively, which here are assumed to be subsets of ordered Euclidean spaces, each with a non-empty closed positive cone $K$. The positive cone $K$ defines an order, and is assumed to have the following properties: it is a cone, i.e. $\alpha K \subset K$ for $\alpha \in \mathbb{R}_{+}$, it is convex and $K \cap(-K)=\{0\}$. An I/O system is then monotone if the output map $h$ is order preserving and for all initial states $x_{1}, x_{2}$ and all inputs $u_{1}(t), u_{2}(t)$ the following property holds: if $x_{1} \preceq x_{2}$ and $u_{1}(t) \preceq u_{2}(t)$ then $\varphi\left(t, x_{1}, u_{1}\right) \preceq \varphi\left(t, x_{2}, u_{2}\right)$ for all $t>0$. Here we will only consider partial orders induced by orthants $K$ in $\mathbb{R}^{n}$ meaning that $x \preceq y$ iff $y-x \in K$.

We can rewrite equations (1)-(4) as an interconnection of two subsystems, which will facilitate the direct application of the small gain theorem. Define system $\Sigma_{1}$ as: 


$$
\left\{\begin{aligned}
\frac{d\left[r I_{2}\right](t)}{d t} & =k_{p}\left[T_{21}^{t o t}\right] \frac{\left[r A_{1}\right](t-\tau)^{n}}{K_{A}^{n}+\left[r A_{1}\right](t-\tau)^{n}}-k_{d}\left[r I_{2}\right](t) \\
& =f_{1}\left(r I_{2}, r A_{1}\right) \\
y_{1}(t) & =[r I 2](t)=h_{1}\left(\left[r I_{2}\right]\right)
\end{aligned}\right.
$$

and system $\Sigma_{2}$ as:

$$
\left\{\begin{aligned}
\frac{d\left[r A_{1}\right](t)}{d t} & =k_{p}\left[T_{12}^{t o t}\right] \frac{K_{I}^{n}}{K_{I}^{n}+[r I 2](t-\tau)^{n}}-k_{d}\left[r A_{1}\right](t) \\
& =f_{2}\left(r A_{1}, r I_{2}\right) \\
y_{2}(t) & =\left[r A_{1}\right](t)=h_{2}([r A 1]) .
\end{aligned}\right.
$$

The systems $\Sigma_{1}$ and $\Sigma_{2}$ are cascaded in a closed-loop system. To apply the small gain theorem [2] we need to verify the following points:

(i) $\Sigma_{1}$ is monotone when its input and output are ordered according to the "standard order", which is the order induced by the positive real semi-axis.

(ii) $\Sigma_{2}$ is monotone when its input is ordered according to the "standard order" and the output uses an order induced by the negative real semi-axis.

(iii) Each subsystem I/O characteristics are respectively monotonically increasing and decreasing.

(iv) Every solution of the closed-loop system is bounded.

(v) The discrete time dynamical system $u_{k+1}=\left(k_{y_{2}} \circ\right.$ $\left.k_{y_{1}}\right)\left(u_{k}\right)$ has a unique globally attractive equilibrium.

First, note that the delays do not affect the stability analysis using the small gain theorem, so from now on they will be neglected [1], [3]. For the system $\Sigma_{1}$ let the input, output and state space $U_{1}, Y_{1}$ and $X_{1}$ respectively be equal to $\mathbb{R}_{+}$with the natural order induced by $\mathbb{R}_{+}$and for the system $\Sigma_{2}$ let the input, output and state space $U_{2}, Y_{2}$ and $X_{2}$ respectively be equal to $\mathbb{R}_{+}$with the natural order induced by $\mathbb{R}_{+}$for $U_{2}$ and $X_{2}$ and with the reverse order induced by $\mathbb{R}_{-}$for $Y_{2}$. In fact $0 \leq\left[r I_{2}\right] \leq \frac{k_{p}}{k_{d}}\left[T_{21}\right]^{\text {tot }}$ and $0 \leq\left[r A_{1}\right] \leq \frac{k_{p}}{k_{d}}\left[T_{12}\right]^{\text {tot }}$ which means that the closed loop system is bounded, i.e. condition $(i v)$ is fulfilled.

Monotonicity of system $\Sigma_{1}$ holds because $\frac{\partial f_{1}}{\partial\left[r A_{1}\right]} \geq 0$ and $h_{1}$ is monotonically increasing [2]. After making a change of variable in system $\Sigma_{2}$ according to $\left[\widetilde{r A}_{1}\right]=-\left[r A_{1}\right]$ it can be seen that $\frac{\partial \tilde{f}_{2}}{\partial\left[r I_{2}\right]} \geq 0$ and $\tilde{h}_{2}$ is monotonically decreasing, where $\tilde{f}_{2}$ and $\tilde{h}_{2}$ are the functions after the change of variable, which shows monotonicity. That means that conditions $(i)-(i i)$ hold. Condition $($ iii $)$ is fulfilled since the I/O characteristic $k_{y_{1}}\left(\left[r A_{1}\right]\right)=\frac{k_{p}}{k_{d}} T_{21}^{t o t} \frac{\left[r A_{1}\right]^{n}}{K_{A}^{n}+\left[r A_{1}\right]^{n}}$ of system $\Sigma_{1}$ is monotonically increasing and the I/O characteristic $k_{y_{2}}\left(\left[r I_{2}\right]\right)=\frac{k_{p}}{k_{d}} T_{12}^{t o t} \frac{K_{I}^{n}}{K_{I}^{n}+\left[r I_{2}\right]^{n}}$ of system $\Sigma_{2}$ is monotonically decreasing.

To prove the existence of a globally attractive equilibrium of the discrete time dynamical system $u_{k+1}=\left(k_{y_{2}} \circ\right.$ $\left.k_{y_{1}}\right)\left(u_{k}\right)$, the Banach fixed point theorem can be applied. Assume that $(X, d)$ is a non-empty complete metric space and let $T: X \rightarrow X$ be a contraction mapping on $X$, i.e. there is a non-negative real number $q<1$ such that $d(T x, T y) \leq q d(x, y)$ for all $x, y$ in $X$. Then the Banach fixed point theorem states that the map $T$ admits one and only one fixed point $x^{*}$ in $X$ and the sequence $x_{n}=T x_{n-1}$ converges to the limit $x^{*}$.
For the oscillator, let $X=R_{+}, d$ be the Euclidean distance and the map $T$ be the composition of the two I/O characteristics $k_{y_{1}}\left(u_{1}\right)=\frac{k_{p}}{k_{d}}\left[T 21^{t o t}\right] \frac{u_{1}^{n}}{K_{A}^{n}+u_{n}^{n}} \equiv k_{1} \frac{u_{1}^{n}}{K_{A}^{n}+u_{1}^{n}}$ and $k_{y_{2}}\left(u_{2}\right)=\frac{k_{p}}{k_{d}}\left[T 12^{t o t}\right] \frac{K_{I}^{n}}{K_{I}^{n}+u_{2}^{n}} \equiv k_{2} \frac{K_{I}^{n}}{K_{I}^{n}+u_{2}^{n}}$, i.e. $T=k_{y_{2}} \circ$ $k_{y_{1}}$. To show that $T$ is a contraction mapping, it is enough to show that the derivative $\left|D\left(k_{y_{2}} \circ k_{y_{1}}\right)\right|<1$. To that end, use the fact that $\left|D\left(k_{y_{2}} \circ k_{y_{1}}\right)(u)\right|=\left|D k_{y_{2}}\left(k_{y_{1}}(u)\right) D k_{y_{1}}(u)\right| \leq$ $\sup \left|D k_{y_{2}}\right| \sup \left|D k_{y_{1}}\right|$. The derivatives are given by $D k_{y_{2}}(u)=-n u^{n-1} k_{2} \frac{K_{I}^{n}}{\left(K_{I}^{n}+u^{n}\right)^{2}}$ and $D k_{y_{1}}(u)=$ $k_{1} \frac{n u^{n-1}}{K_{A}^{n}+u^{n}}-n u^{n-1} k_{1} \frac{u^{n}}{\left(K_{A}^{n}+u^{n}\right)^{2}}$ respectively and the suprema are found at $\arg \sup \left|D k_{y_{2}}\right|=K_{I}\left(\frac{n-1}{n+1}\right)^{1 / n} \equiv K_{I} c$ and $\arg \sup \left|D k_{y_{1}}\right|=K_{A}\left(\frac{n-1}{n+1}\right)^{1 / n} \equiv K_{A} c$ respectively, where $c$ is introduced to simplify calculations. This makes the suprema of the derivatives equal to $\sup \left|D k_{y_{2}}\right|=\left|D k_{y_{2}}\left(K_{I} c\right)\right|=\frac{k_{2}}{K_{I}} \frac{n c^{n-1}}{\left(1+c^{n}\right)^{2}}$ and $\sup \left|D k_{y_{1}}\right|=$ $\left|D k_{y_{1}}\left(K_{A} c\right)\right|=\frac{k_{1}}{K_{A}} \frac{n c^{n-1}}{\left(1+c^{n}\right)^{2}}$. Restricting ourselves to the case when $k_{1}=k_{2}=k$ and $K_{I}=K_{A}=K$ the derivative of the map $T$ becomes bounded by $\mid D\left(k_{y_{2}} \circ\right.$ $\left.k_{y_{1}}\right)(u)|\leq \sup | D k_{y_{2}}|\sup | D k_{y_{1}} \mid=\left(\frac{k}{K}\right)^{2}\left(\frac{n c^{n-1}}{\left(1+c^{n}\right)^{2}}\right)^{2}$. The last inequality is less than one when $\frac{K}{k}>\frac{n c^{n-1}}{\left(1+c^{n}\right)^{2}}$. With $n=5$ the condition for convergence is $\frac{K}{k} \gtrsim 1.3$.

This relationship between the thresholds $K=K_{A}=K_{I}$ and the ratio of production to degradation rates $k=\frac{k_{p}}{k_{d}}\left[T_{i j}^{t o t}\right]$ defines a region in the parameter space where oscillations are not admitted.

\section{B. Describing functions}

The describing function (DF) method is an approximate technique allowing to determine whether a closed loop linear dynamical system presenting nonlinearities can admit periodic orbits. This technique has been previously applied to models of biological oscillators [10], investigating the effect of delays, of the Hill coefficient and of the linear transfer function order. The DF method can be used to determine at what frequencies periodic solutions can occur, what the amplitude and offset are, and whether the oscillatory regime is stable or not.

The idea with describing functions is that they give the gain of the system at a particular frequency just as the transfer function does for linear systems. The difference is that describing functions are dependent on the size of the input signal, which is natural since they describe a nonlinear system. To define the describing functions for the dc-component and fundamental frequency of a static nonlinear system $f$, assume that the input signal can be written as $w(t)=B+A \sin (\omega t)$. The output of the static system equals $z(t)=f(w(t)) \approx$ $c_{0}(A, B)+c_{1}(A, B) \sin (\omega t+\phi(A, B))$, where the last step is a truncation of the Fourier series of the output. It then follows that the first two describing functions can be written as $H(A, B)=\frac{c_{0}(A, B)}{B}$ and $F(A, B)=\frac{c_{1}(A, B) e^{i \phi(A, B)}}{A}$ respectively.

The method can be directly applied to the biochemical oscillator described by equations (1)-(4). The dynamical 


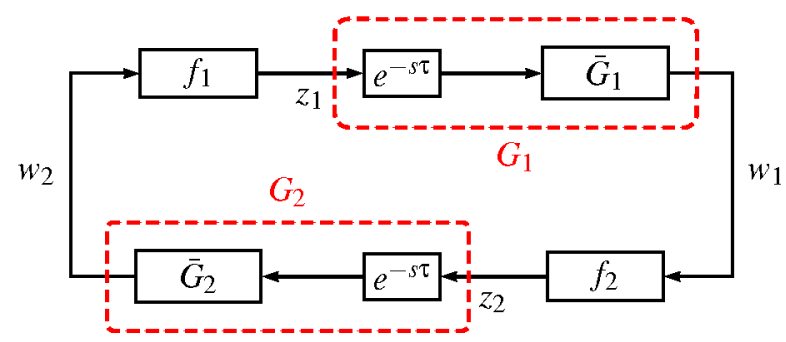

Fig. 2: Block diagram of the biochemical oscillator. Each $\bar{G}_{i}$ block represents a linear dynamical system, while the $f_{i}$ blocks represent a static nonlinearity.

system can be viewed as a closed-loop system with two subsystems, each consisting of a low-pass filter $\bar{G}_{i}(s)=$ $\frac{k_{p}}{s+k_{a}}$ with a delay $e^{-s \tau}$, cascaded with the static nonlinear Hill function $\left[T_{21}^{t o t}\right] \frac{\left[r A_{1}\right]^{n}}{K_{A}^{n}+\left[r A_{1}\right]^{n}}$ and $\left[T_{12}^{t o t}\right] \frac{K_{I}^{n}}{K_{I}^{n}+\left[r I_{2}\right](t-\tau)^{n}}$ respectively (Fig. 2). The presence of low pass transfer functions guarantees that higher frequencies will be attenuated, satisfying one of the requirements for the application of the DF method. Indeed, the harmonic balance technique is only verified at the zero and first order harmonics, neglecting higher frequency components of the propagated signal.

The harmonic balance equations that have to be solved are [4], [10]:

$$
\begin{aligned}
& \prod_{j=1}^{2} H_{j}\left(A_{j}, B_{j}\right) G_{j}(0)=1, \\
& \prod_{j=1}^{2} F_{j}\left(A_{j}, B_{j}\right) G_{j}(i \omega)=1,
\end{aligned}
$$

assuming that the input signals to the two nonlinearities can be written as $w_{j}(t)=B_{j}+A_{j} \sin (\omega t), j \in\{1,2\}$ and that $H_{j}$ and $F_{j}$ are the so called describing functions for the dc-component and fundamental frequency respectively. Equations (6)-(7) express the requirement for sustained oscillations, which is that the closed loop gain for the offset component and fundamental frequency has to be equal to one.

\section{Numerical results}

In this section, the area of the parameter space admitting oscillations will be analyzed numerically, comparing the results with the small gain theorem predictions. Such numerical analysis is based on the DF method, solving equations (6)(7). After rewriting $A_{2}$ and $B_{2}$ in terms of $A_{1}, B_{1}$ and $\omega$, this becomes a system of three unknowns, which can be solved with the MATLAB function lsqnonlin with constraints enforcing the variables to be positive. In fact $\omega$ can be solved for separately from equation (7), which comes from the fact that the describing functions $F_{j}$ are real for the model of the biochemical oscillator. This leaves two unknowns. To ensure that the periodic solutions are stable, we need to verify that the derivative of the closed-loop gain for the fundamental frequency as a function of the amplitude $A_{1}$ is negative at the amplitude $A_{1, s o l}$ of the oscillations [10]. This ensures stability because if the amplitude is slightly increased

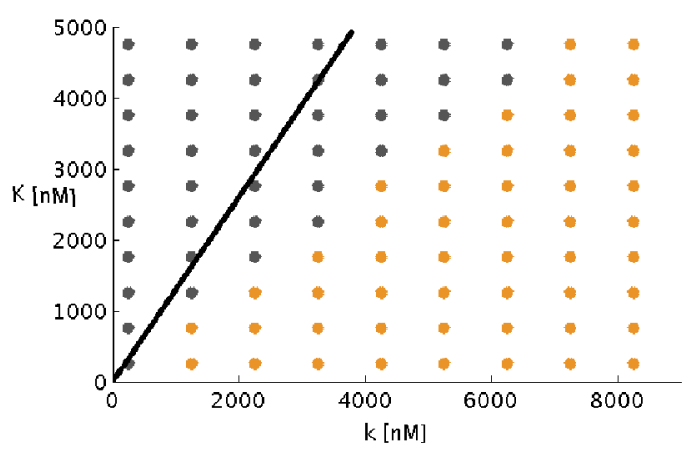

Fig. 3: Simulation results based on the DF method, illustrating the influence of the parameters $K$ and $k$ on the oscillatory regime. Orange dots indicate the existence of stable oscillations and gray means a lack thereof. The limit given by the small gain theorem is represented by the black line, to the left of which the system is asymptotically stable.

then the closed-loop gain will decrease and the trajectory will return to the limit cycle. The converse holds if the amplitude is decreased. To determine whether the derivative is negative, two amplitudes $A_{1, \text { left }}=A_{1, \text { sol }}-\triangle A_{1}$ and $A_{1, \text { right }}=A_{1, \text { sol }}+\triangle A_{1}$, where $\triangle A_{1}$ is small, are chosen. Then $B_{1}\left(A_{1}\right)$ is solved for in equation (6) and finally the two closed-loop gains $\prod_{j=1}^{2} F_{j}\left(A_{j}, B_{j}\right) G_{j}(i \omega)$ with $A_{1}=A_{1, \text { left }}$, $B_{1}=B_{1}\left(A_{1, \text { left }}\right)$ and $A_{1}=A_{1, \text { right }}, B_{1}=B_{1}\left(A_{1, \text { right }}\right)$ respectively are calculated. By taking the difference of the gains, the sign of the derivative is obtained.

The small gain theorem gave us a relation between $k=$ $\frac{k_{p}}{k_{d}}\left[T_{i j}^{t o t}\right]$ and $K=K_{A}=K_{I}$ when the system is globally asymptotically stable. This relation can be verified with the describing function analysis by marking whether the system displays stable oscillations or not in a graph with $k$ on the $\mathrm{x}$-axis and $K$ on the $\mathrm{y}$-axis (Fig. 3). The describing function analysis also provides additional information for parameter values where global asymptotic stability is not achieved.

The quotient $k=\frac{k_{p}}{k_{d}}\left[T_{i j}^{t o t}\right]$ that appears in the small gain theorem analysis indicates the importance of having the right relation between $k_{p}$, which can be interpreted as the concentration of the enzyme RNAP and $k_{d}$, which can be interpreted as the concentration of the enzyme RNase $\mathrm{H}$. Having too little RNAP or too much RNase $\mathrm{H}$ will make the quotient $\frac{K}{k}>1.3$, which implies that there can not be sustainable oscillations.

To highlight the effects of the choice of enzyme concentrations, the frequency and amplitude of the oscillations can be plotted as a function of $k_{p}$ and $k_{d}$ by applying the describing function technique (Fig. 4). Once again it can be seen that if the quotient $\frac{k_{p}}{k_{i}}$ gets too small oscillations are not achieved, but it is also seen that $k_{d}$ being too small prohibits oscillations.

Last it is interesting to answer whether a too high $k_{p}$ or in other words too much of the enzyme RNAP, has negative effects. It turns out that such a choice results in oscillations with increasingly longer periods. Using only the 

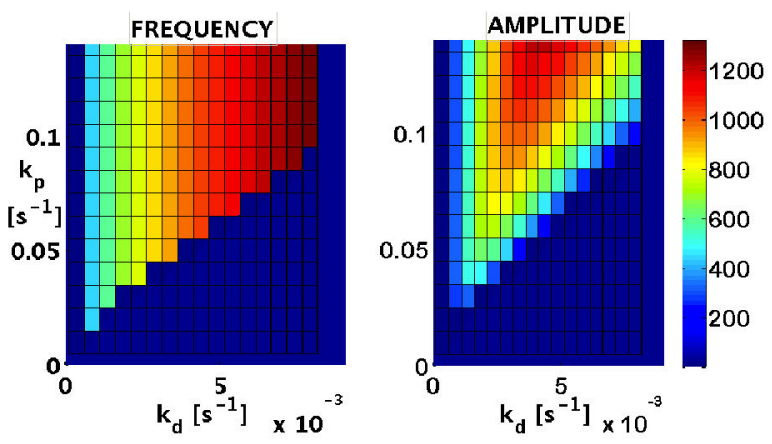

Fig. 4: Amplitude and frequency are plotted as a function of of $k_{p}$ (RNAP) and $k_{d}$ (RNase H). The system does not achieve oscillations in the dark blue areas.

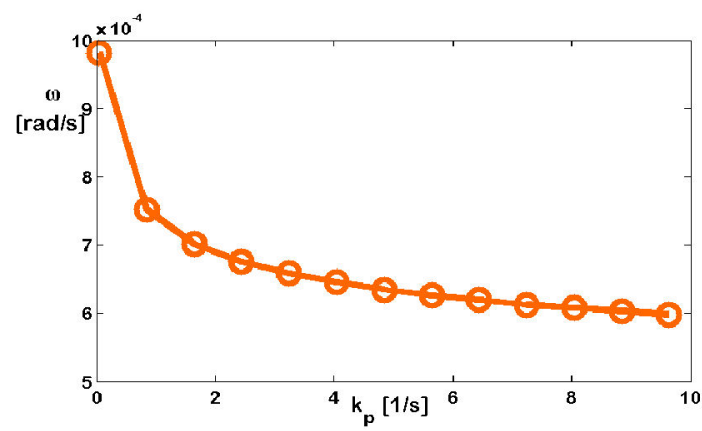

Fig. 5: Numerical simulation of the angular frequency plotted versus $k_{p}$ with $\tau=1200 \mathrm{~s}$. A high value of RNA production $k_{p}$ results in slow oscillations.

MATLAB function dde 23 in combination with a program that detects the extrema of the trajectory, it is possible to generate a graph with the angular frequency plotted versus $k_{p}$ (Fig. 5). It is clear from the graph that the frequency decreases monotonically with $k_{p}$ which is the fact that makes it undesirable to have too much RNAP. When periods start approaching several hours, it is no longer practical for real experiments. To emphasize this effect, the delay $\tau$ was here also increased to $1200 \mathrm{~s}$. Regardless of the choice of $\tau$ the monotonically decreasing graphs are obtained, but with this particular choice the period becomes as high as 3 hours when $k_{p}=9.6 s^{-1}$.

\section{EXPERIMENTAL RESULTS}

The analysis based on the small gain theorem and the DF method was helpful in directing the experimental work on the synthetic oscillator. Previous observations on the role of the enzyme amounts suggested that frequency and amplitude of the oscillations were particularly sensitive to such parameters. In particular, Figure 4 shows that only certain ratios of RNA production (RNAP) to degradation (RNase $\mathrm{H}$ ) will yield an oscillatory regime. We decided to focus on this aspect of the numerical results, and run a series of experiments where the enzyme volumes were systematically tuned. The results qualitatively confirm the numerical predictions and provide a methodology to modulate frequency and amplitude of the oscillations.

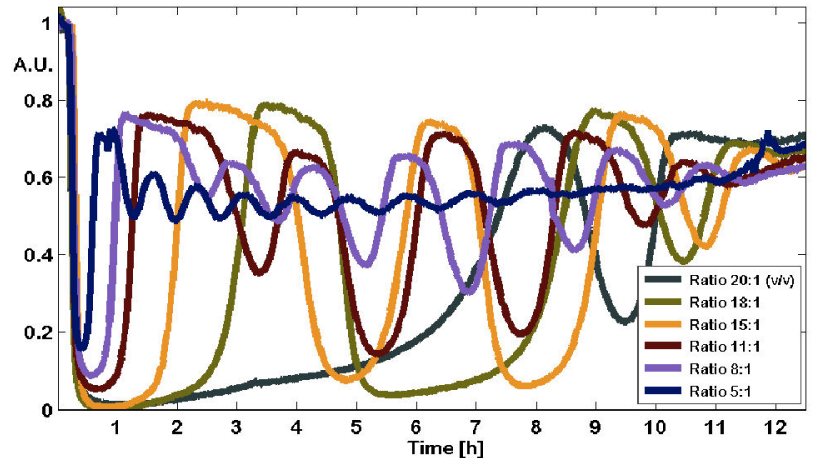

Fig. 6: Effects of RNAP: RNase $\mathrm{H}$ volume ratio for a fixed total enzyme volume of $6 \mu \mathrm{L}$. The oscillatory regime is lost for ratios that are too low (the period decreases until the high amplitude is damped) or too high (the period increases while the amplitude is shrinked).

1) Materials and Methods: All the DNA was purchased from Integrated DNA Technologies, Coralville, IA. Template $T_{21}$ is monitored in the experiments, and is labeled with the TYE665 fluorophore $(649 / 665 \mathrm{~nm}$ ex/em), while Activator $d A 1$ is labeled with the IAB fq quencher molecule. The strand sequences are the same as those used for the two-node oscillator in Chapter 5 of [5], Section 5.3. T7 RNAP was purchased from Epicentre Biotechnologies, Cat. n. TM910K. E. coli cloned RNase $\mathrm{H}$ was purchased from Ambion, Cat. $\mathrm{n}$. AM2292. Nucleoside triphosphates (NTPs) were purchased from Epicentre Biotechnologies, Cat. n. RN02825. Transcription buffer and dithiothreitol (DTT) were purchased from Epicentre Biotechnologies, Cat. n. BP1001. Pyrophosphatase was purchased from Sigma Aldrich, Cat. n. I1891-100UN, and diluted in Tris $\mathrm{HCl} 20 \mathrm{mM}, \mathrm{pH} 7.2,50 \%$ glycerol (v/v) to a final concentration of 0.015 Units $/ \mu \mathrm{L}$.

The DNA templates were annealed by heating at $95^{\circ} \mathrm{C}$ and slow cooling cycles. Each experiment had the target DNA concentrations of $T_{12} 60 \mathrm{nM}, d A 1250 \mathrm{nM}, T_{21} 125 \mathrm{nM}$, $d A 1175 \mathrm{nM}$ and $d I 1350 \mathrm{nM}$. The buffer conditions were set at: $7.5 \mathrm{mM}$ each NTP, $1 x$ transcription buffer, $10 \mathrm{mM}$ DTT, $35 \mathrm{mM}$ Magnesium Chloride, $0.015 \mathrm{U} / \mu \mathrm{L}$ pyrophosphatase. Final volume was of $60 \mu \mathrm{L}$. The total enzyme amounts in solution were varied between $2-10$ Units for RNase $H$ and $400-1400$ Units for RNAP. The unit definitions for each enzyme used are provided by the vendors.

The on/off state of the template $T_{21}$ was monitored by measuring the fluorescence of the corresponding fluorophore. When the template is bound to its activator, i.e. the gene is on, its fluorescence is quenched. The signal is instead high when the gene is off. The experiments were run at $37^{\circ} \mathrm{C}$ in a Fluorolog 3 Spectrofluorimeter, Horiba Jobin-Yvon. The data are normalized to arbitrary units between 0 and 1 , relative to maximum and minimum fluorescence measured for the $T_{21}$ switch.

2) Results: If the volume ratio of RNAP to RNase $H$ is varied, a range of oscillatory trajectories is observed. The system can sustain oscillations for 12-18 hours: the loss of 


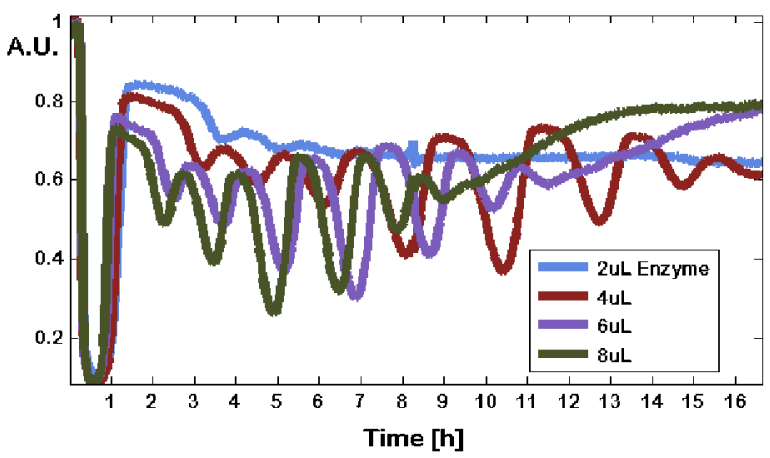

Fig. 7: Effects of total enzyme volume variations for a fixed RNAP: RNase $\mathrm{H}$ volume ratio of $8: 1$. The oscillatory regime is lost if the total volume is too low, because the production/degradation dynamics are not sufficiently sustained.

enzyme activity, build up of waste products and exhaustion of NTPs cause the degradation of the oscillatory performance over time. For a total enzyme volume fixed at $6 \mu \mathrm{L}$, with the RNAP:RNase $H$ ratio varied between $20: 1$ and $5: 1$, the results are shown in Figure 6. If the enzyme ratio is fixed to $8: 1$, while the total volume is varied, we obtain results as in Figure 7. While we cannot explicitly map the enzyme amounts that are used in experiments with the phenomenological parameters $k_{p}$ and $k_{d}$, the theoretical analysis is qualitatively confirmed by the experiments. We can reason in terms of transcription and degradation "strength". In Figure 4, we observe that for a given $k_{p}$, or transcription strength, oscillations are achieved if the degradation is within certain bounds. We can repeat a similar reasoning if we fix $k_{d}$. In Figure 6 we observe that indeed sustained oscillations (presenting period between 1 and 3 hours, and amplitude more than 0.3) are obtained only for enzyme ratios between $18: 1$ and $8: 1$. On the other hand, for a given ratio, varying the total volume of enzyme can result in reaching or losing the oscillatory regime. In Figure 7, for a ratio of $8: 1$, oscillations are lost for a too low total amount of enzyme. Qualitatively, the plot in Figure 4 also suggests that if $k_{p}$ and $k_{d}$ are both low, the oscillations vanish. The results showing the overall influence of enzyme amounts on amplitude and frequency of oscillations is summarized in Figure 8, which is the experimental counterpart of Figure 4. As predicted in the numerical study of the effects of $k_{p}$ on the oscillations period (Fig. 5), when the amount of RNAP is too high, we obtain oscillations with very low frequency and large amplitude. Conversely, when a low enzyme ratio is used, the system hits a regime presenting very fast and low amplitude oscillations. These plots show how by solely tuning the enzyme amounts we can modulate frequency and amplitude, but the two seem to be inversely correlated and cannot be set independently.

\section{ConClusions}

In this paper we have considered an in vitro genetic circuit given by a two node oscillator. Such a system has been recently proposed [7]. Given the cost and duration of the

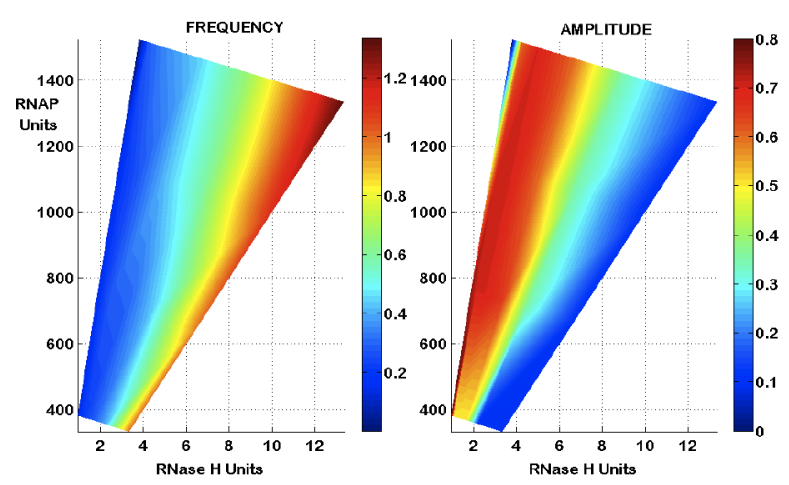

Fig. 8: The data collected varying enzyme ratios and volumes are summarized and fitted. The areas of the graph that are not in color correspond to enzyme amounts that will not yield an oscillatory regime.

experiments, having a predictive qualitative model of the system is helpful in exploring the oscillator performance. Rather than resorting solely to extensive numerical simulation of the full chemical reaction network composing the oscillator, we focused our attention on a simplified phenomenological model. The application of the small gain theorem and the method of the describing functions allowed us to derive simple relationships between the system parameters that characterize convergence of the system rather than a periodic behavior. Further numerical analysis showed how production and degradation rates define areas in the parameter space where oscillations are possible. These results were helpful in guiding the experiments on this system, and were qualitatively confirmed by the data collected.

\section{REFERENCES}

[1] Angeli, D. and Sontag, E.D. Oscillations in I/O Monotone Systems Under Negative Feedback IEEE Transactions on Automatic Control, $2008,53,166-176$

[2] Angeli, D. and Sontag, E.D. Monotone Control Systems Arxiv preprint math, 2002

[3] Angeli, D. and Sontag, E.D Interconnections of monotone systems with steady-state characteristics Lecture Notes in Control and Information Sciences, 2004, Springer, 135-154

[4] Davison, E. and Constantinescu, D. A describing function technique for multiple nonlinearities in a single-loop feedback system IEEE Trans. on Automatic Control, 1971, 16, 56-60

[5] Kim, J. In vitro synthetic transcriptional networks $\mathrm{PhD}$ Thesis, California Institute of Technology, 2006

[6] Kim, J., White, K. S. and Winfree, E. Construction of an in vitro bistable circuit from synthetic transcriptional switches Molecular Systems Biology, 2006, 68

[7] Kim, J. and Winfree, E. Synthetic In Vitro Transcriptional Oscillators In preparation.

[8] Markham, N. R. and Zuker, M. DINAMelt web server for nucleic acid melting prediction Nucleic Acids Res., 2005, 33, 577-581

[9] Nucleic Acid Package: http://www.nupack.org

[10] Rapp, P. Mathematical Techniques for the Study of Oscillations in Biochemical Control Loops Bull. Inst. Math. Appl, 1976, 12, 11-20

[11] Seelig, G., Soloveichik, D., Zhang, D. Y. and Winfree, E. Enzyme-free nucleic acid logic circuits Science, 2006, 314, 1585-1588

[12] Simmel, F. DNA nanodevices Small, 2005, 1, 284-299

[13] Yurke, B. and Mills, A. P. Using DNA to Power Nanostructures. Genetic Programming and Evolvable Machines, 2003, 4, 111-122 